\title{
Identification and Analysis of MicroRNAs Associated with Wing Polyphenism in the Brown Planthopper, Nilaparvata lugens
}

\author{
Le Xu ${ }^{1,+}{ }^{D}$, Jiao Zhang ${ }^{2,+}$, Anran Zhan ${ }^{1,+}$, Yaqin Wang ${ }^{3}$, Xingzhou Ma ${ }^{1,2}$, Wencai Jie ${ }^{2}$, \\ Zhenghong Cao ${ }^{1}$, Mohamed A. A. Omar ${ }^{1,4}\left(\mathbb{D}, \mathrm{Kang} \mathrm{He}^{1, * \mathbb{D}}\right.$ and Fei Li ${ }^{1}$ (D) \\ 1 State Key Laboratory of Rice Biology \& Ministry of Agricultural and Rural Affairs Key Laboratory of \\ Molecular Biology of Crop Pathogens and Insect Pests, Institute of Insect Sciences, Zhejiang University, \\ Hangzhou 310058, China; xule1208@zju.edu.cn (L.X.); zhananran@zju.edu.cn (A.Z.); \\ zxmlmq@163.com (X.M.); 3130100574@zju.edu.cn (Z.C.); dr.abdelwanees@alexu.edu.eg (M.A.A.O.); \\ lifei18@zju.edu.cn (F.L.) \\ 2 College of Plant Protection, Nanjing Agricultural University, Nanjing 210095, China; \\ 15064210267@139.com (J.Z.); jiewencai@gmail.com (W.J.) \\ 3 State Key Laboratory of Rice Biology, Institute of Biotechnology, Zhejiang University, \\ Hangzhou 310058, China; wangyq0219@126.com \\ 4 Department of Plant Protection, Faculty of Agriculture (Saba Basha), Alexandria University, \\ Alexandria 21531, Egypt \\ * Correspondence: hekang@zju.edu.cn \\ $\dagger$ These authors contributed equally to this work.
}

Received: 19 November 2020; Accepted: 14 December 2020; Published: 21 December 2020

\begin{abstract}
Many insects are capable of developing two types of wings (i.e., wing polyphenism) to adapt to various environments. Though the roles of microRNAs (miRNAs) in regulating animal growth and development have been well studied, their potential roles in modulating wing polyphenism remain largely elusive. To identify wing polyphenism-related miRNAs, we isolated small RNAs from 1st to 5th instar nymphs of long-wing (LW) and short-wing (SW) strains of the brown planthopper (BPH), Nilaparvata lugens. Small RNA libraries were then constructed and sequenced, yielding 158 conserved and 96 novel miRNAs. Among these, 122 miRNAs were differentially expressed between the two BPH strains. Specifically, 47, 2, 27 and 41 miRNAs were more highly expressed in the 1st, 3rd, 4th and 5th instars, respectively, of the LW strain compared with the SW strain. In contrast, 47, 3, 29 and 25 miRNAs were more highly expressed in the 1st, 3rd, 4th and 5th instars, respectively, of the SW strain compared with the LW strain. Next, we predicted the targets of these miRNAs and carried out Gene Ontology and Kyoto Encyclopedia of Genes and Genomes pathway analysis. We found that a number of pathways might be involved in wing form determination, such as the insulin, MAPK, mTOR, FoxO and thyroid hormone signaling pathways and the thyroid hormone synthesis pathway. Thirty and 45 differentially expressed miRNAs targeted genes in the insulin signaling and insect hormone biosynthesis pathways, respectively, which are related to wing dimorphism. Among these miRNAs, Nlu-miR-14-3p, Nlu-miR-9a-5p and Nlu-miR-315-5p, were confirmed to interact with insulin receptors (NlInRs) in dual luciferase reporter assays. These discoveries are helpful for understanding the miRNA-mediated regulatory mechanism of wing polyphenism in BPHs and shed new light on how insects respond to environmental cues through developmental plasticity.
\end{abstract}

Keywords: microRNA; wing polyphenism; insulin; hormone 


\section{Introduction}

Polyphenism, which occurs when organisms share the same genotype but exhibit two or more distinct phenotypes, is crucial for organisms to successfully deal with heterogeneous environments. This ecological phenomenon may be determined by multiple environmental conditions, including temperature, diet and population density [1]. For instance, the southern African butterfly, Bicyclus anynana, develops into a dry season form when exposed to a low temperature during the larval stage [2] and female honeybees, depending on the composition of nutrients they are fed as larvae, develop into two different castes, exhibiting a large degree of variation in morphology, physiology and social function [3,4]. Depending on the local population density, locusts transform between two phases, a low-density "solitarious" phase and a high-density "gregarious" phase $[5,6]$. Wing polyphenism is well known in insects, with distinct wing phenotypes conferring differential dispersal abilities [7-9]. Long-wing (LW) individuals with fully developed wings and flight muscles are capable of dispersing to new habitats, while short-wing (SW) or wingless individuals allocate resources to reproduction rather than to flight $[1,10]$.

The brown planthopper (BPH), Nilaparvata lugens (stål) (Hemiptera: Delphacidae) shows obvious wing polyphenism in response to environmental cues [10]. This capability enables the BPH to migrate over long distances and because of this, it has become a notorious rice pest in Asia. The insulin/insulin-like growth factor (IGF) signaling (IIS) pathway participates in the determination of wing morphs in BPHs [11]. Specifically, two insulin receptors, InR1 and InR2, play opposing roles in controlling the development of wings by regulating the activity of the master transcription factor, FoxO [11,12]. Wounding and host quality also affect the development of wing morphs via regulation of the IIS pathway $[13,14]$. With high-quality host plants, nymphs will develop into the SW form through the induction of Ultrabithorax (NlUbx) expression [15]. Moreover, insect hormones, including juvenile hormones (JHs) and ecdysone, also act as important factors in wing-morph determination [10]. High titers of $\mathrm{JH}$ in the penultimate nymphal instar induce $\mathrm{BPH}$ nymphs to develop into SW adults [16], while silencing JH epoxide hydrolase (Nliheh), a metabolic enzyme catalyzing $\mathrm{JH}$ degradation, increases the ratio of SW individuals in the population [16]. As a key endocrine signal, ecdysone modulates insect molting and metamorphosis but also participates in wing morph determination in the aphid Acyrthosiphon pisum [17,18]. Multiple signaling pathways are involved in regulating wing morph determination and dozens of genes show differences in stage-dependent expression between LW and SW BPHs [19]. However, how these genes work together in the associated pathways to modulate polyphenism is still unknown.

MicroRNAs (miRNAs) are small non-coding RNA molecules $(\sim 22 \mathrm{nt})$ that function in posttranscriptional repression of gene expression in diverse eukaryotic lineages [20]. Numerous studies have shown that miRNAs play roles in a complex regulatory system for biological processes [21-26]. In insects, miRNAs are involved in the regulation of growth, metamorphosis and reproduction [27-32]. For instance, multiple miRNAs jointly regulate the metamorphosis development via the biosynthesis of 20E in Chilo suppressalis [27]. Additionally, miRNAs also function in the phenotypic plasticity. In the locust, miR-133 inhibition resulted in the behavioral shift from the solitary phase to the gregarious phase [30]. Overexpression of $m i R-9 b$ facilitated the development of wing and reduced the proportion of winged offspring in aphids [31]. Recently, Nlu-miR-34 was found to mediate cross talk between the IIS pathway and two hormones, JH and 20-Hydroxyecdysone (20E), by targeting InR1 and forming a positive feedback loop [32]. Given that multiple factors regulate wing polyphenism in $\mathrm{BPH}$, we hypothesize that miRNAs can indirectly control wing morph plasticity by regulating these key determinants, including factors involved in signal transduction pathways and hormone biosynthesis. Here, we constructed and sequenced small RNA libraries of nymph BPHs at the 1st to 5th instar stages for both the LW and SW strains. Differentially expressed miRNAs between the two strains were identified and the targets of these miRNAs were predicted. Gene Ontology (GO) and Kyoto Encyclopedia of Genes and Genomes (KEGG) pathway enrichment analyses were performed to annotate the functions and pathways involved in the regulation of wing polyphenism. Some miRNAs 
were also selected to validate the interactions with targets. Our results reveal a complicated miRNA network that potentially determines the stage-dependent modulation of wing morph plasticity in BPH. This network increases our understanding of how miRNA regulates wing polyphenism and should be helpful for developing novel methods for controlling $\mathrm{BPH}$ in the future.

\section{Results}

\subsection{Identification of miRNAs in BPH}

To identify miRNAs associated with the development of wing morph plasticity, we first pooled 20 small RNA libraries constructed with RNA isolated from 1st to 5th instar nymphs of LW and SW BPH strains with two replicates per sample. After removing the low-quality sequences, 148,129,963 and 160,959,695 clean reads were obtained for the LW and SW strains, respectively (Table 1). The distributions of total small RNA read lengths were similar with a distinct bimodal peak (one peak at 21-23 nt and another at 26-29 nt) in both strains (Figure 1). To identify and annotate the full repertoire of miRNAs in BPH, we used two different algorithms, miRDeep2 [33,34] and MapMi [35], to predict conserved and novel miRNAs. In total, we identified 254 miRNAs (158 conserved and 96 novel miRNAs) in the small RNA libraries, which were combined before miRNA prediction (Supplementary Table S1). The conserved miRNAs belong to 60 known miRNA families, while the novel miRNAs belong to 42 unknown miRNA families. The number of miRNAs identified here is consistent with the number identified in BPH in previous studies [36,37] and these miRNAs were used for further analysis.

Table 1. Reads obtained from small RNA libraries sequenced in Nilaparvata lugens.

\begin{tabular}{|c|c|c|c|c|c|c|c|c|c|}
\hline No & Sample & $\begin{array}{l}\text { Raw } \\
\text { Reads }\end{array}$ & $\begin{array}{c}\text { High } \\
\text { Quality }\end{array}$ & $\begin{array}{l}\text { Clean } \\
\text { Reads }\end{array}$ & Q20 & $\begin{array}{c}\text { GC } \\
\text { Content }\end{array}$ & $\begin{array}{c}5 \text { 'adapter } \\
\text { Contamines }\end{array}$ & $\begin{array}{l}3^{\prime} \text { adapter_Null } \\
\text { or Insert_Null }\end{array}$ & $\begin{array}{c}\text { with } \\
\text { PloyA/T/G/C }\end{array}$ \\
\hline 1 & LW-1st-1 & $13,625,214$ & $13,614,741$ & $13,330,526$ & $98.25 \%$ & $48.45 \%$ & 3519 & 217,585 & 62,872 \\
\hline 2 & LW-1st-2 & $14,390,780$ & $14,380,460$ & $14,079,327$ & $98.33 \%$ & $47.56 \%$ & 2826 & 234,820 & 63,230 \\
\hline 3 & LW-2nd-1 & $13,447,515$ & $13,437,862$ & $13,204,811$ & $98.25 \%$ & $47.64 \%$ & 2869 & 162,612 & 67,300 \\
\hline 4 & LW-2nd-2 & $12,552,925$ & $12,544,080$ & $12,284,953$ & $98.22 \%$ & $47.33 \%$ & 2611 & 189,043 & 67,244 \\
\hline 5 & LW-3rd-1 & $14,008,106$ & $13,997,085$ & $13,723,894$ & $98.40 \%$ & $47.89 \%$ & 3182 & 194,273 & 75,452 \\
\hline 6 & LW-3rd-2 & $13,190,404$ & $13,178,303$ & $12,975,678$ & $98.22 \%$ & $48.60 \%$ & 3609 & 149,545 & 49,216 \\
\hline 7 & LW-4th-1 & $18,344,628$ & $18,328,565$ & $17,708,977$ & $98.13 \%$ & $47.87 \%$ & 5209 & 540,722 & 73,320 \\
\hline 8 & LW-4th-2 & $18,656,643$ & $18,637,940$ & $17,960,139$ & $98.11 \%$ & $48.57 \%$ & 4572 & 603,621 & 69,249 \\
\hline 9 & LW-5th-1 & $17,176,623$ & $17,165,563$ & $16,607,021$ & $98.32 \%$ & $47.57 \%$ & 5806 & 474,728 & 77,696 \\
\hline 10 & LW-5th-2 & $16,751,585$ & $16,739,158$ & $16,254,637$ & $98.12 \%$ & $47.58 \%$ & 5980 & 419,723 & 58,517 \\
\hline 11 & SW-1st-1 & $17,257,604$ & $17,247,057$ & $16,612,101$ & $98.16 \%$ & $47.29 \%$ & 8058 & 505,257 & 121,319 \\
\hline 12 & SW-1st-2 & $13,757,659$ & $13,748,950$ & $13,172,776$ & $98.21 \%$ & $46.95 \%$ & 5890 & 466,836 & 103,188 \\
\hline 13 & SW-2nd-1 & $15,251,104$ & $15,240,012$ & $14,434,758$ & $98.28 \%$ & $47.38 \%$ & 3383 & 683,287 & 118,280 \\
\hline 14 & SW-2nd-2 & $19,765,649$ & $19,750,938$ & $19,257,968$ & $98.33 \%$ & $47.36 \%$ & 4397 & 344,244 & 143,995 \\
\hline 15 & SW-3rd-1 & $18,780,777$ & $18,766,573$ & $17,960,404$ & $98.41 \%$ & $48.60 \%$ & 5290 & 696,703 & 103,821 \\
\hline 16 & SW-3rd-2 & $16,793,873$ & $16,779,788$ & $16,026,154$ & $98.26 \%$ & $48.51 \%$ & 4287 & 666,676 & 82,349 \\
\hline 17 & SW-4th-1 & $13,125,238$ & $13,116,371$ & $12,854,273$ & $98.26 \%$ & $48.00 \%$ & 7247 & 142,684 & 111,947 \\
\hline 18 & SW-4th-2 & $13,552,267$ & $13,541,969$ & $13,173,223$ & $98.12 \%$ & $47.65 \%$ & 4728 & 229,093 & 134,671 \\
\hline 19 & SW-5th-1 & $19,598,809$ & $19,582,736$ & $18,883,495$ & $98.21 \%$ & $47.47 \%$ & 4252 & 567,450 & 127,157 \\
\hline 20 & SW-5th-2 & $19,297,940$ & $19,282,397$ & $18,584,543$ & $98.29 \%$ & $47.92 \%$ & 4342 & 573,153 & 120,010 \\
\hline
\end{tabular}

LW: long-wing strain, SW: short-wing strain, High quality: raw reads without low quality reads, Q20: the proportion of bases with phred value $>20,5^{\prime}$ adapter contamines: reads with 5'adapter, 3' adapter_null or insert_null: reads without $3^{\prime}$ adapter or insertion, with ployA/T/G/C: reads with ployA/T/G/C. 


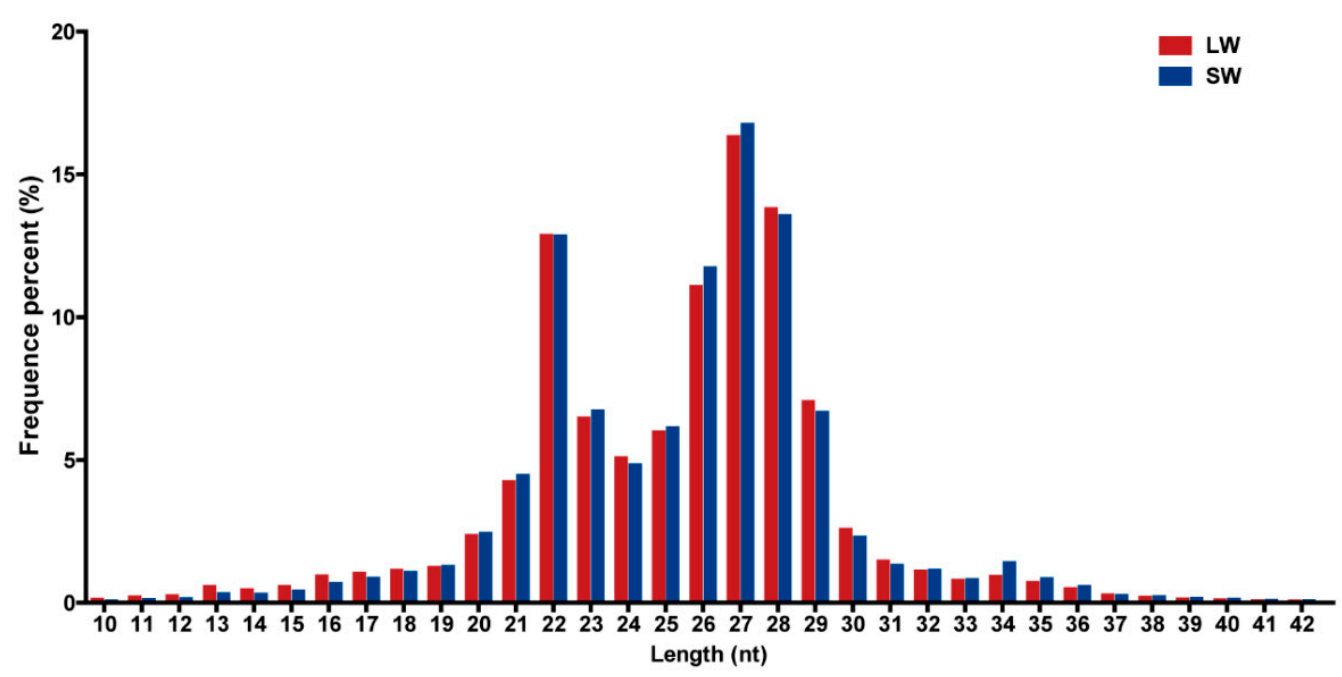

Figure 1. Distribution of total small RNA read lengths in long-wing (LW) and short-wing (SW) brown planthopper (BPH) strains.

\subsection{Identification of Wing Morph-Related miRNAs in BPH}

To investigate the effect of miRNAs on the regulation of wing morph plasticity, we first calculated the reads counts for each miRNA and then used DESeq2 [38] to compare miRNA expression in the LW and SW BPH strains at the same stage (Supplementary Table S2). A total of 122 miRNAs were identified as being differentially expressed between the two strains during nymph development (Figure 2A and Supplementary Table S3). Among them, 94 miRNAs were differentially expressed in the 1st instar, including 47 miRNAs more highly expressed in LW strain and 47 miRNAs more highly expressed in the SW strain. In the 4th instar nymph, 27 miRNAs were more highly expressed in the LW strain while 29 miRNAs were more highly expressed in the SW strain. In the 5th instar, 41 miRNAs were more highly expressed in the LW strain, while only 25 miRNAs were more highly expressed in the SW strain. Moreover, in the 3rd instar, only two miRNAs were more highly expressed in the LW strain and three miRNAs were more highly expressed in the SW strain. No miRNAs were differentially expressed in the 2nd instar (Figure 2B).

In particular, Nlu-nov-5-5p was significantly more highly expressed in all four instar stages of LW BPHs, while Nlu-nov-26-5p showed a more complicated expression pattern, with a high level of expression in the 1st and 4th instar stages of the SW strain and high expression in the 3rd and 5th instar stages of the LW strain (Supplementary Figure S1). Interestingly, 13 miRNAs were significantly more highly expressed in the 1st and 4th instar stages but significantly more lowly expressed in 5th instar stage of the LW strain compared with the SW strain. However, another 19 miRNAs showed a similar expression pattern (i.e., higher expression in the 1st and 4th instar but lower expression in the 5th instar) when comparing the SW strain with the LW strain. The presence of these differentially expressed miRNAs implies that miRNAs are probably associated with the regulation of wing plasticity in $\mathrm{BPH}$. 


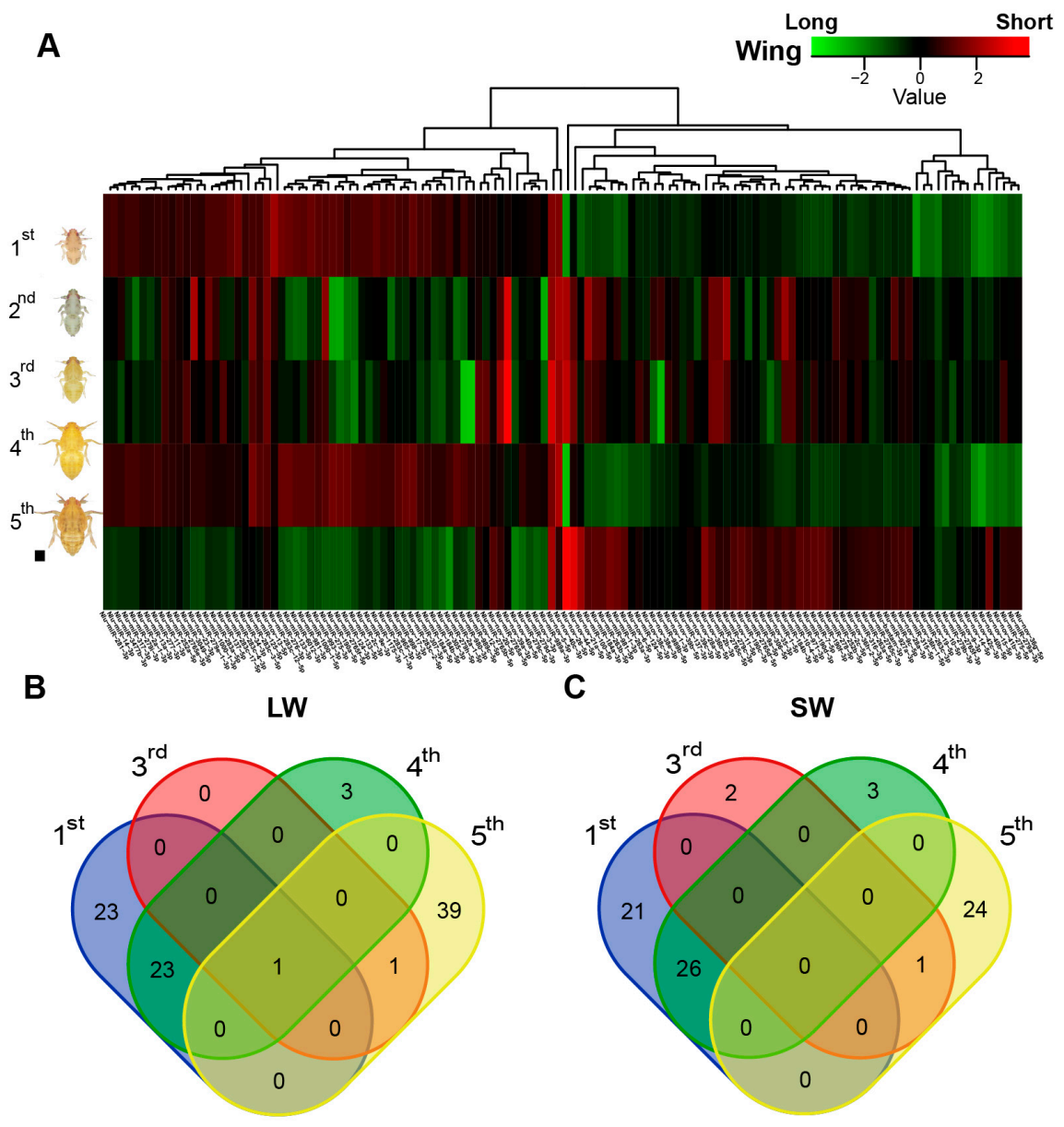

Figure 2. Differentially expressed miRNAs between LW and SW strains. (A) Heatmap of differentially expressed miRNAs between LW and SW strains. Green indicates miRNAs more highly expressed in the LW strain. Red indicates miRNAs more highly expressed in the SW strain. $(\mathbf{B}, \mathbf{C})$ Venn diagrams showing the overlap of miRNAs more highly expressed in the LW (B) and SW (C) strains at different instar stages (Differential expression analysis by DESeq2, adjusted $p$-value $<0.05$, fold change $>2$ ).

\subsection{Functional Analysis of Differentially Expressed miRNAs}

To further investigate the roles of these differentially expressed miRNAs, we used four algorithms, miRanda [39], TargetScan [40], RNAhybrid [41,42] and PITA [43], to predict the miRNA targets. In total, 1907 and 1724 genes were predicted as targets of the miRNAs more highly expressed in the LW and SW strains, respectively (Supplementary Tables S4 and S5). In both the LW and SW BPH strains, the predicted target genes were mainly annotated to GO terms related to binding activity, such as protein binding, carbohydrate derivative binding, ion binding, ubiquitin protein ligase binding and nucleosideand purine-related binding activities (Figure 3A and Supplementary Tables S6 and S7). In particular, miRNAs more highly expressed in LW strain BPH also participated in the regulation of protein activities, including nucleoside-triphosphatase activity (GO:0017111, adjusted $p$-value $=2.66 \times 10^{-5}$ ), pyrophosphatase activity (GO:0016462, adjusted $p$-value $\left.=4.13 \times 10^{-5}\right)$, primary active transmembrane transporter activity (GO:0015399, adjusted $p$-value $\left.=1.32 \times 10^{-4}\right)$ and ATPase activity (GO:0016887, adjusted $p$-value $\left.=1.44 \times 10^{-4}\right)($ Supplementary Tables S6 and S7).

KEGG pathway enrichment analysis showed that target genes of all the miRNAs more highly expressed in the LW strain were significantly enriched in 32 pathways, including the insulin signaling pathway $($ KO04910, adjusted $p$-value $=0.0017)$, MAPK signaling pathway (KO04013, adjusted $p$-value $=0.017)$, mTOR signaling pathway $(K O 04150$, adjusted $p$-value $=0.028)$, FoxO signaling pathway $(\mathrm{KO} 04068$, adjusted $p$-value $=0.030)$, thyroid hormone signaling pathway (KO04919, 
adjusted $p$-value $=0.018)$ and thyroid hormone synthesis $($ KO04918, adjusted $p$-value $=0.024)$ (Figure 3B and Supplementary Table S8). Only 10 pathways were significantly enriched for the targets of all the miRNAs more highly expressed in the SW strain and all were among the 32 enriched pathways in the LW strain (Figure 3B and Supplementary Table S9). Notably, targets of differentially expressed miRNAs in both strains included those involved in the endocrine system (insulin signaling pathway and gonadotropin-releasing hormone $(\mathrm{GnRH})$ signaling) and signal transduction (PI3K-Akt signaling pathway and MAPK signaling pathway), implying their vital roles in the regulation of wing morph plasticity. Moreover, some specific pathways only enriched in the LW strain are speculated to be responsible for the development of winged morphs; for example, the Hippo signaling pathway (KO04390, adjusted $p$-value $=0.050$ ) was found to participate in morphogen control of wing growth in Drosophila [44-46]. Interestingly, the insulin signal transduction pathway was only significantly enriched in the 5th instar nymph of the LW strain (KO04910, adjusted $p$-value $=5.89 \times 10^{-3}$ ); however, insulin signaling (KO04910, adjusted $p$-value $=9.71 \times 10^{-3}$ in the 1 st instar and $1.63 \times 10^{-2}$ in the 4th instar) and related transduction pathways such as the PI3K-Akt signaling pathway $\left(\right.$ KO04151, adjusted $p$-value $=9.71 \times 10^{-3}$ in the 4 th instar $)$ and MAPK signaling pathway (KO04013, adjusted $p$-value $=3.03 \times 10^{-2}$ in the 1 st instar and $1.63 \times 10^{-2}$ in the 4 th instar) were enriched in specific nymph stages of the SW strain (Supplementary Tables S10 and S11). Moreover, thyroid hormone synthesis (KO04918, adjusted $p$-value $=1.33 \times 10^{-2}$ ) was only significantly enriched in the 5 th instar of the LW strain and was also identified as significantly enriched in the targets of highly expressed miRNAs from all LW stages. This indicates that miRNAs participate in the modulation of wing type plasticity via insulin signal transduction and that there is complicated endocrine regulation at different stages of BPH development.

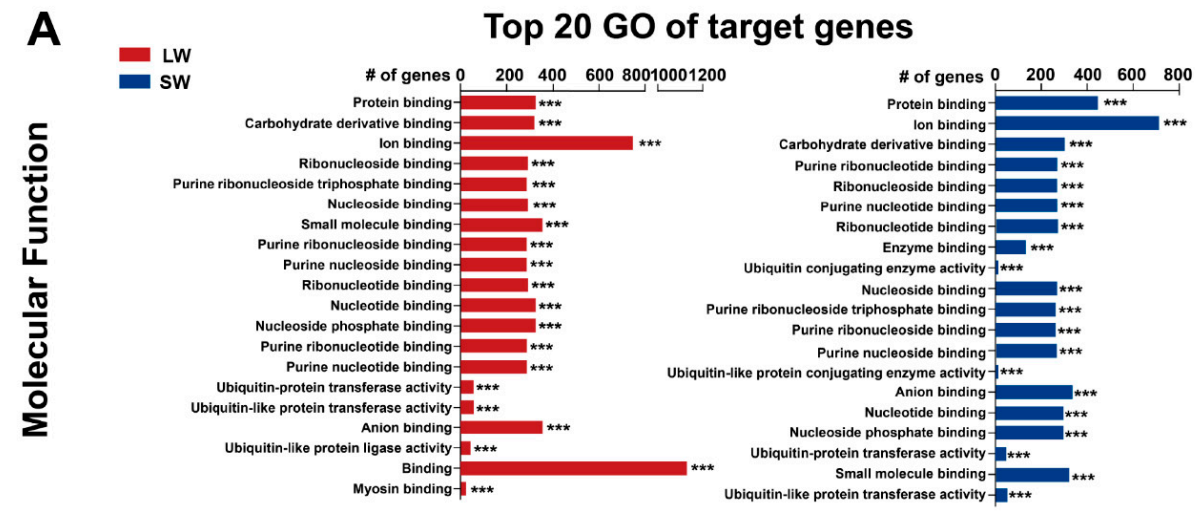

B

\section{Top 20 KEGG pathway of target genes}
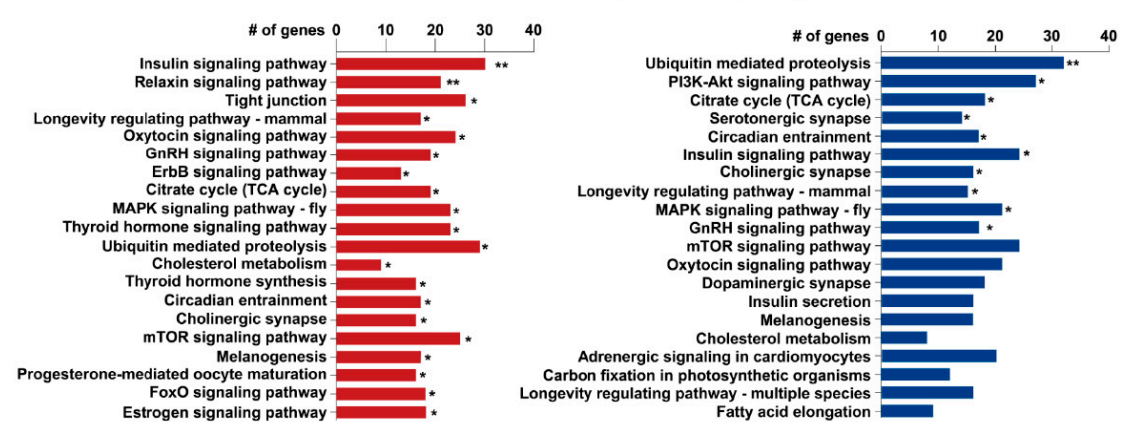

Figure 3. Gene Ontology (GO) and Kyoto Encyclopedia of Genes and Genomes (KEGG) enrichment analyses of targets of differentially expressed miRNAs. (A) GO molecular function terms enriched in differentially expressed miRNA-targeted genes in LW and SW strains. (B) KEGG pathways enriched in differentially expressed miRNA-targeted genes in LW and SW strains. ${ }^{*} p<0.05,{ }^{* *} p<0.01,{ }^{* * *} p<0.001$. 


\subsection{Validation of miRNAs and Target Gene}

To further validate the interaction of miRNAs and target genes involved in insulin signal transduction pathways, we first searched for the target genes of all the differentially expressed miRNAs in this pathway. Since insect hormone-related genes are involved in insect development and particularly in wing polymorphism in insects [47-51], the complete sequences of these genes were also included in the target input file. We found that 26 miRNAs shared more than one target gene and that 22 miRNAs had target genes in both the insulin and insect hormone pathways. The broad-complex core protein $(B r-C)$ gene, which is an upstream factor in the insulin signaling pathway [32], was targeted by as many as 21 miRNAs and the insulin receptor (NIInR) genes were targeted by 13 miRNAs (Figure 4 and Supplementary Table S12). Since insulin receptors control wing plasticity in BPH [11], three miRNAs, Nlu-miR-14-3p, Nlu-miR-9a-5p and Nlu-miR-315-5p, which are predicted to target the insulin receptors, were selected to confirm the interaction between miRNAs and target genes in dual luciferase reporter assays in vitro. In the presence of miRNA mimic, luciferase activities decreased significantly compared with those of the negative control (Figure 5). These results indicate that these miRNAs might modulate the wing morph of BPHs via the insulin signaling pathway and insect hormone regulation.

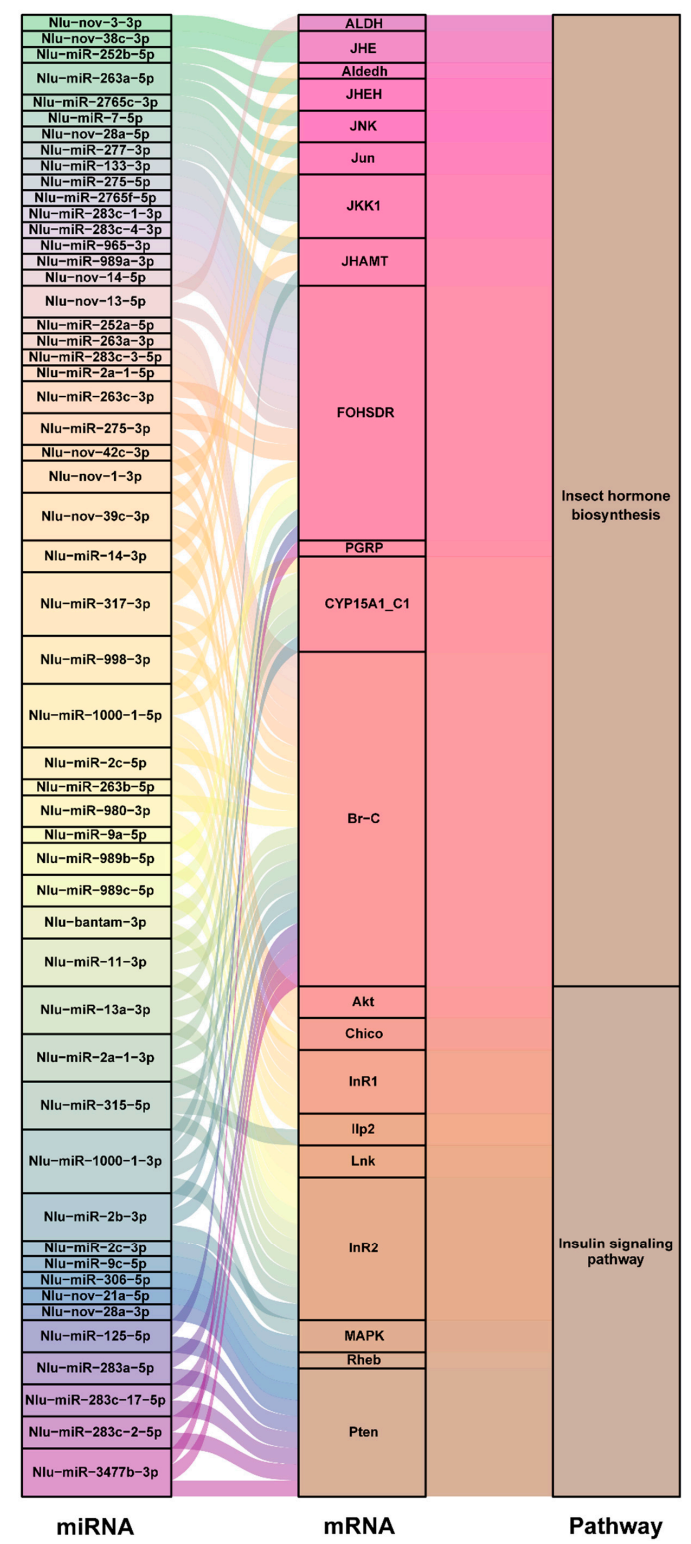

Figure 4. Sankey diagrams of miRNAs, target genes and enriched pathways predicted by more 
than two algorithms (miRanda, TargetScan, RNAhybrid and PITA). ALDH, Aldehyde dehydrogenase dimeric NADP preferring; JHE, Juvenile hormone esterase; Aldedh, Aldehyde dehydrogenase cytosolic 2-like; JHEH, Juvenile hormone epoxide hydrolase; JNK, Mitogen-activated protein kinase 8/9/10 (c-Jun N-terminal kinase); JUN, Transcription factor AP-1; JKK1, Dual specificity mitogen-activated protein kinase kinase 7-like; JHAMT, Juvenile hormone-III synthase; FOHSDR, Farnesol dehydrogenase-like; PGRP, Peptidoglycan recognition protein; CYP15A1_C1, Methyl farnesoate epoxidase-like; Br-C, Broad-complex core protein; $A k t$, Serine/threonine-specific protein kinase; Chico, Insulin receptor substrate; InR1, Insulin receptor 1; Ilp2, Insulin-like peptide; Lnk, SH2B adapter protein 1-like protein; InR2, Insulin receptor 2; MAPK, Mitogen-activated protein kinase; Rheb, Ras homolog enriched in brain; Pten, Phosphatidylinositol-3,4,5-trisphosphate 3-phosphatase.
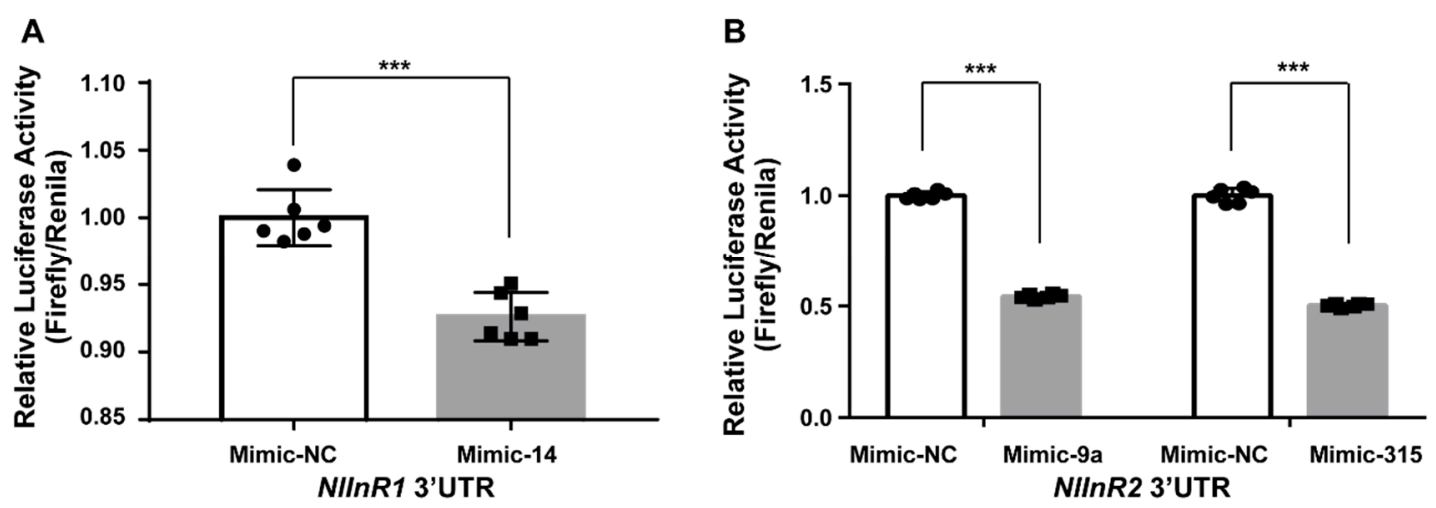

Figure 5. Dual luciferase reporter assays confirmed the interactions between miRNAs and NlInR transcripts in vitro. (A) The interaction of Nlu-miR-14-3p with NIInR1. (B) The interaction of $N l u-m i R-9 a-5 p$ and Nlu-miR-315-5p with NIInR2. In the presence of mimic, the relative luciferase activities were significantly decreased compared with the negative control (NC) group. The black circulars and black squares are presented as 6 replicates. Data are means $\pm \operatorname{SEM}(n=6) .{ }^{* * *} p<0.001$.

\section{Discussion}

Wing polymorphism is a successful strategy allowing BPHs to adapt different environmental factors [10]. Multiple factors play pivotal roles in controlling wing polyphenism, particularly those functioning in the IIS signaling pathway and endocrine biosynthesis in insects [10]. However, whether miRNAs regulate the phenotypic plasticity of the wing via these pathways is seldom investigated. Here, we focused on identifying miRNAs regulating wing determination at different developmental stages of BPHs. By sequencing small RNA libraries, we identified 254 miRNAs, including 158 conserved and 96 novel miRNAs, which is similar to the total number of miRNAs reported in a previous study of BPH [36]. Moreover, we identified 122 miRNAs that were differentially expressed (>2-fold change) between the LW and SW strains in at least one of four nymph instar stages (1st, 3rd, 4th and 5th instars). This suggests that these miRNAs are associated with the regulation of wing polyphenism in BPH. Interestingly, differentially expressed miRNAs in 1st instar shared similar expression pattern to those in 4th instar with the heatmap (Figure 2A). And we also found 24 miRNAs in both two instars significantly highly expressed in LW strain, while 26 miRNAs showed similar pattern in SW strain. Additionally, 22 of 23 other highly expressed miRNAs in 1st instar of LW strain, also highly expressed in 4 th instar with the low fold change or no significant difference ( $p$-value $>0.05$ ) (Supplementary Table S2). In SW strain, 20 of 21 other highly expressed miRNAs in 1st instar were in the same situation (Supplementary Table S2). This result indicates that wing polymorphism in BPH may be regulated via similar miRNAs in these two instars when compared with that in 2nd and 3rd instars. However, the reason for this similar expression pattern is still unclear and it needs further investigation to elucidate the roles of these miRNAs. 
Differentially expressed miRNAs likely regulate wing polymorphism via multiple pathways at different instar stages in these two strains. In the 1st and 4th instar nymphs, targets of highly expressed SW miRNAs were significantly enriched in multiple pathways, such as adrenergic signaling in cardiomyocytes (KO04261), fatty acid metabolism (KO01212), dopaminergic synapse (KO04728) and the pyruvate metabolism pathway (KO00620). Polyunsaturated fatty acids are reported to play crucial roles in insect reproduction $[52,53]$. And in a previous study, a reduction in synaptic vesicular release was associated with Drosophila flight deficits [54]. The lower expression of genes in these pathways may lead to the high reproduction and weak flight ability in SW strain. In contrast, in the 3rd instar nymphs, the targets of miRNAs more highly expressed in the LW strain were significantly enriched in the sphingolipid (KO04071) and HIF-1 (KO04066) signaling pathways. Sphingolipid signaling is involved in insect development via wingless signaling and the sphingosine-1-phosphate lyase homolog Sply regulates the pattern of the dorsal longitudinal flight muscles of the Drosophila adult thorax $[55,56]$. Nymphal miRNAs might regulate the sphingolipid signaling pathway to strengthen the dorsal longitudinal flight muscles allowing for long-distance migration of the LW BPH strain. In the 5th instar nymphs, targets of miRNAs more highly expressed in the LW strain were enriched in multiple pathways, such as the regulation of lipolysis in adipocytes (KO04923), thyroid hormone synthesis (KO04918) and the glucagon signaling pathway (KO04922) and the targets of miRNAs more highly expressed in the SW strain were enriched in the longevity regulating (KO04211), mTOR signaling (KO04150) and glycosphingolipid biosynthesis (KO00604) pathways. In addition, some pathways were differentially regulated between the LW and SW strains at different instar stages. For instance, the MAPK signaling (KO04013) and insulin signaling (KO04910) pathways were significantly enriched in differentially expressed miRNA target genes in 1st and 4th instar SW nymphs and in 5th instar LW nymphs. The results indicated that the expression of miRNAs varies between instars and that miRNAs regulate wing dimorphism of $\mathrm{BPH}$ in a specific and complicated manner by targeting genes at different developmental stages in the SW and LW strains.

Previously, the tight junction pathway (KO04530) was found to be enriched in differentially expressed genes across the egg, nymph and adult stages between LW and SW strain BPHs [19]. In our study, the tight junction pathway was also significantly enriched in the targets of miRNAs highly expressed in the LW strain (adjusted $p$-value $=1.18 \times 10^{-2}$ ) (Supplementary Table S8). Moreover, targets of miRNAs more highly expressed in the LW strain were also enriched in vascular smooth muscle contraction (KO04270, adjusted $p$-value $=3.80 \times 10^{-2}$ ) and the oxytocin signaling pathway $\left(\right.$ KO04921, adjusted $p$-value $\left.=1.62 \times 10^{-2}\right)($ Supplementary Table S8). Zhang et al. [19] also found that differentially expressed genes between LW and SW strain at the 3rd instar nymph stage were significantly enriched in vascular smooth muscle contraction and the oxytocin signaling pathway. These results imply that the regulation of miRNA expression might be involved in the determination of wing length.

Among the differentially expressed miRNAs, 53 were predicted to target numerous genes in the insulin signaling pathway or insect hormone biosynthesis-related pathways and 22 of these miRNAs were predicted to target genes in both pathways (Supplementary Table S12). Therefore, we speculate that miRNAs mediate crosstalk between the IIS pathway and insect hormones to modulate the development of wing morphs. Although Nlu-miR-34 was not included in the list of differentially expressed miRNAs because it only showed a 1.7-fold change in expression between the LW and SW $\mathrm{BPH}$ strains at the whole-body level, the expression in wing buds may be higher in the SW strain. This implies that tissue-specific expression of miRNAs might play a vital role in regulating the $\mathrm{JH}$, 20E and IIS pathways at key stages where wing morph is determined-the 2nd to 4th instar nymph stages [32]. A dual luciferase reporter assay confirmed the interaction of NIInR with three miRNAs, Nlu-miR-14-3p, Nlu-miR-9a-5p and Nlu-miR-315-5p, in vitro; however, further evidence from in vivo overexpression or knock-down experiments are needed to clarify the functions of these miRNAs in the regulation of wing plasticity. 
In previous study, Yang et al. [57] found that miR-1000 regulates morphogenesis of the wing and compound eyes in the American cockroach. The fact that we identified Ultrabithorax (NlUbx, MK855103.1) as a potential target of the differentially expressed miRNA Nlu-miR-1000-1-3p, which regulates wing dimorphism by regulating host nutrition status [15], provides evidence that Nlu-miR-1000 plays an important role in wing polyphenism. In addition, the targets of differentially expressed miRNAs in the LW strain were also significantly enriched in the Hippo signaling pathway, which is regarded as a conserved regulator of organ size in Drosophila [46,58]. Nlu-miR-263a-5p is predicted to target protein expanded, which is a component of the Hippo signaling pathway (Supplementary Tables S4 and S5). Protein expanded is a mediator between fat and the Hippo signaling pathway and fat participates in morphogen control of wing growth of Drosophila [44-46]. This indicates that Nlu-miR-263a-5p may regulate wing development polymorphism by targeting protein expanded.

\section{Materials and Methods}

\subsection{Insect Rearing}

The BPHs were originally collected in rice fields of Wuhan, Hubei Province, China and maintained in a climate room at $26 \pm 1{ }^{\circ} \mathrm{C}$ under a $16 \mathrm{~L}: 8 \mathrm{D}$ light regime with a relative humidity of $75 \pm 5 \%$. New seedlings of the susceptible rice variety Taichuang Native 1 (TN1) were used for feeding. To obtain the LW and SW strains, in each generation LW and SW individuals from the LW and SW populations, respectively, were selected at the adult stage as described in a previous study [59] and selection was carried out for more than 50 generations. The rates of LW and SW BPHs in the corresponding strains used for experiments were at least $80 \%$.

\subsection{Small RNA Library Preparation and Sequencing}

The whole body of BPH nymphs from the 1st to 5th instars (day 1 of each stage) were collected for small RNA sequencing with two biological replicates. Total RNA was extracted with TRIzol. The RNA degradation and contamination were monitored on $1 \%$ agarose gels. The purity, concentration and integrity of RNA were tested by NanoPhotometer spectrophotometer (IMPLEN, Los Angeles, USA), Qubit 2.0 fluorometer (Life Technologies, Carlsbad, USA) and Agilent Bioanalyzer 2100 system (Agilent Technologies, Palo Alto, USA), respectively. $3 \mu \mathrm{g}$ RNA per sample was used as input to generate the sequencing libraries using the NEBNext Multiplex Small RNA Library Prep Kit for Illumina (NEB, Ipswich, USA) following the manufacturer's protocol. After ligating the $3^{\prime}$ SR adaptor and $5^{\prime}$ SR adaptor to the ends of the small RNAs, M-MuLV Reverse Transcriptase (RNase $\mathrm{H}^{-}$) was used to synthesize the first strand cDNA. Then, PCR amplification was performed and the products were purified by electrophoresis on an $8 \%$ polyacrylamide gel at $100 \mathrm{~V}$ for $80 \mathrm{~min}$. Fragments of $140-160 \mathrm{bp}$ were recovered and the library quality was assessed using DNA High Sensitivity Chips (Agilent Technologies, Palo Alto, USA) on the Agilent Bioanalyzer 2100 system. The library preparations were sequenced at Novogene (Beijing, China) on the Illumina HiSeq 2500/2000 platform.

\section{3. miRNA Prediction}

To identify miRNAs, adaptors and low-quality reads were first removed from the raw data for each small RNA library, then the clean data for all the libraries were combined together and analyzed using two algorithms, miRDeep2 [33,34] and MapMi [35]. For miRDeep2, mapper.pl was used to map the reads to the reference genome (parameters: $-\mathrm{c}-\mathrm{j}-118-\mathrm{m}-\mathrm{p}$ BPH $-\mathrm{s}-\mathrm{t}-\mathrm{v}$; discarding reads shorter than $18 \mathrm{nt}$ ). Conserved and novel miRNAs were identified by miRDeep2.pl with the miRBase mature sequence [60] (the minimum cut-off score was set by 5 and significant Randfold $p$-value is set as yes). MapMi was performed with the cut-off score more than 25 and minimum free energy (MFE) less than $-18 \mathrm{kcal} / \mathrm{mol}$. The high quality BPH genome sequence (accession number GCA_014356525.1) [61] was used as a reference. miRNAs predicted by both algorithms were retained for further analysis after 
removing redundant sequences. After comparing to the miRNAs in miRBase, unmatched miRNA candidates were filtered with the criterion described in miRBase [60] and then were identified as novel miRNAs.

\subsection{Differential Expression Analysis}

To identify miRNAs differentially expressed in each library, miRNA frequencies were calculated using a Perl script after removing the miRNAs with a low number of reads (total count for all samples $<50$ ). In the same instar of each strain, the miRNA counts of two libraries were compared by R package ggplot2 (Supplementary Figure S2) and it indicated that the expression of miRNAs was highly reproducible between biological replicates. Then the pairwise comparison of the filtered data was analyzed using the R package DESeq2 with a standard workflow [38]. Only those miRNAs with greater than a 2-fold change (FC $>2$ ) in expression and a significant false discovery rate (adjusted $p$-value $<0.05$ ) were determined to be significantly differentially expressed between the corresponding nymph stages of the two strains.

\subsection{Target Gene Prediction and GO and KEGG Enrichment Analysis}

To predict the target genes of differentially expressed miRNAs, $3^{\prime}$ untranslated region (UTR) sequences of BPH were downloaded from InsectBase [62]. Complete sequences of genes in BPH wing polymorphism-related pathways such as the insect hormone biosynthesis pathway (KO00981) and insulin signaling pathway (KO04910) were identified and downloaded from the National Center for Biotechnology Information (NCBI, Bethesda, USA) (Supplementary Table S13). Next, four algorithms, miRanda [39], TargetScan [40], RNAhybrid [41,42] and PITA [43] were used for miRNA prediction. The energy minimum was set at $<-20 \mathrm{kcal} / \mathrm{mol}$ for prediction by miRanda, while targets filtered with $p$-value $<0.05$ were retained by RNAhybrid. TargetScan and PITA were run with the default parameters. The miRNA-target pairs predicted by all four algorithms were kept for further analysis. The target genes of differentially expressed miRNAs were annotated to GO terms using Blast2GO software [63] and mapped to KEGG pathways using the BlastKOALA web server (https://www.kegg.jp/blastkoala/). NCBI non-redundant protein sequences database was used in Blast2GO to annotate GO number of miRNA targets with blastx configuration. In BlastKOALA web server, miRNA targets were blasted with animal group datasets. With the results of GO number and KEGG number of targets, the enrichment analysis was analyzed and visualized on the cloud platform of OmicShare (http: $/ /$ www.omicshare.com/tools). The background of enrichment is the whole annotation genes in BPH. For each strain, we combined the target genes of highly expressed miRNAs among four instars and performed strain specific enrichment of GO terms and KEGG pathways (Supplementary Tables S6-S9). For highly expressed miRNAs in each instar stage, the KEGG pathways enrichment of their target genes was also analyzed (Supplementary Tables S10 and S11). All results were filtered with the adjusted $p$-value $\leq 0.05$.

\subsection{In Vitro Luciferase Assays}

The 3'UTR sequences of the target genes NIInR1 and NIInR2 were cloned downstream of the firefly luciferase gene in the pMIR-REPORT vector (Obio, Shanghai, China). The Renilla luciferase gene in the pRL-CMV vector (Promega, Madison, USA) was used as a control luciferase reporter. HEK293T cells (Obio, Shanghai, China) were cultured for $24 \mathrm{~h}$ in 96-well culture plates with DMEM (Gibco, Carlsbad, USA) and $10 \%$ FBS (Hyclone, Logan, USA) at $37^{\circ} \mathrm{C}$ and $5 \% \mathrm{CO}_{2}$. miRNA mimics (RiboBio, Guangzhou, China) were added to Lipofectamine 2000 (Invitrogen, Carlsbad, USA) at a concentration of $100 \mathrm{nM}$ for transfection. A random sequence miRNA mimic, UUUGUACUACACAAAAGUACUG, was used as the negative control. Cells were transfected with a transfection reagent mixture of PMIR-REPORT: pRL-CMV: Lipofectamine 2000 at a ratio of $0.2 \mu \mathrm{g}$ : $0.01 \mu \mathrm{g}: 0.25 \mu \mathrm{L}$ following the manufacturer's instructions. At $48 \mathrm{~h}$ post transfection, the activities of the two luciferase enzymes were measured on an Infinite M1000 (Tecan, Männedorf, Switzerland) with six replicates. The ratio of the mean 
firefly luciferase activity to mean Renilla luciferase activity (Firefly/Renilla) was calculated to obtain the relative luciferase activity and ratios were compared using the two-tailed Student's $t$-test.

\section{Conclusions}

The significance of miRNAs function in wing polymorphism will continue to grow as more genes and pathways identified to regulate the development of wing morphs. In this work, we constructed small RNA libraries of all nymph instars of two BPH strains with different wing types and revealed a complex association of miRNAs and their regulatory network ensuring the development of distinct wing morphs between the LW and SW strains of BPH. miRNAs differentially expressed at specific stages regulate wing polyphenism mainly via two mechanisms, the regulation of gene expression levels and hormone titers. For gene expression level, miRNAs regulate the wing morphs via the insulin signaling pathway and some other pathways related to the flight ability (like sphingolipid signaling pathway), the reproduction ability (like dopaminergic synapse pathway). For hormone titers, miRNAs could function in the biosynthesis of JH, 20E and other hormones in insects. The expression of miRNAs varies between instars but 1 st and 4 th instars shared similar expression pattern. We will analyze the relationship of these two instars in future. And regulation of miRNAs in wing dimorphism is also complicated and specific at different developmental stages in two strains. These findings provide a candidate gene set, which will be helpful for mining the key factors involved in the determination of wing plasticity.

Supplementary Materials: The following are available online at http://www.mdpi.com/1422-0067/21/24/9754/s1, Supplementary materials are uploaded.

Author Contributions: F.L. conceived the project and designed the research; L.X., X.M. and W.J. conducted the bioinformatic analysis; J.Z., A.Z. and Z.C. carried out the experiments; L.X., K.H., M.A.A.O. and F.L. discussed the data; L.X. drafted the manuscript; K.H., F.L., Y.W. and L.X. improved the manuscript. All authors have read and agreed to the published version of the manuscript.

Funding: This study is supported by the National Natural Science Foundation of China $(31972354,31701785)$, the Fundamental Research Funds for the Central Universities (2020QNA6024) and 2019 Zhejiang University Academic Award for Outstanding Doctoral Candidates (2019065).

Conflicts of Interest: The authors declare no conflict of interest.

\section{Abbreviations}

$\begin{array}{ll}\text { LW } & \text { Long-wing } \\ \text { SW } & \text { Short-wing } \\ \text { GO } & \text { Gene Ontology } \\ \text { KEGG } & \text { Kyoto Encyclopedia of Genes and Genomes } \\ \text { BPH } & \text { Brown planthopper } \\ \text { NCBI } & \text { National Center for Biotechnology Information } \\ \text { Akt } & \text { Serine/threonine-specific protein kinase } \\ \text { Aldedh } & \text { Aldehyde dehydrogenase cytosolic 2-like } \\ \text { ALDH } & \text { Aldehyde dehydrogenase dimeric NADP preferring } \\ \text { Br-C } & \text { Broad-complex core protein } \\ \text { Chico } & \text { Insulin receptor substrate } \\ \text { CYP15A1_C1 } & \text { Methyl farnesoate epoxidase-like } \\ \text { FOHSDR } & \text { Farnesol dehydrogenase-like } \\ \text { GnRH } & \text { Gonadotropin-releasing hormone } \\ \text { IIS } & \text { Insulin/insulin-like growth factor signaling } \\ \text { Ilp2 } & \text { Insulin-like peptide } \\ \text { InR1 } & \text { Insulin receptor 1 } \\ \text { InR2 } & \text { Insulin receptor 2 } \\ \text { JH } & \text { Juvenile hormone } \\ \text { JHAMT } & \text { Juvenile hormone-III synthase } \\ \text { JHE } & \text { Juvenile hormone esterase }\end{array}$




$\begin{array}{ll}\text { JHEH } & \text { Juvenile hormone epoxide hydrolase } \\ \text { JKK1 } & \text { Dual specificity mitogen-activated protein kinase kinase 7-like } \\ \text { JNK } & \text { Mitogen-activated protein kinase 8/9/10 (c-Jun N-terminal kinase) } \\ \text { JUN } & \text { Transcription factor AP-1 } \\ \text { Lnk } & \text { SH2B adapter protein 1-like protein } \\ \text { MAPK } & \text { Mitogen-activated protein kinase } \\ \text { PGRP } & \text { Peptidoglycan recognition protein } \\ \text { Pten } & \text { Phosphatidylinositol-3,4,5-trisphosphate 3-phosphatase } \\ \text { Rheb } & \text { Ras homolog enriched in brain }\end{array}$

\section{References}

1. Simpson, S.J.; Sword, G.A.; Lo, N. Polyphenism in insects. Curr. Biol. 2011, 21, R738-R749. [CrossRef] [PubMed]

2. Brakefield, P.M.; Frankino, W.A. Polyphenisms in Lepidoptera: Multidisciplinary approaches to studies of evolution and development. In Phenotypic Plasticity of Insects: Mechanisms and Consequences, 1st ed.; Whitman, D.W., Ananthakrishnan, T.N., Eds.; CRC Press: Boca Raton, FL, USA, 2009; pp. 337-368.

3. Huber, F. New Observations on the Natural History of Bees, 3rd ed.; Pub. W. \& C. Tait: Edinburgh, UK, 1821.

4. Haydak, M.H. Honey bee nutrition. Annu. Rev. Entomol. 1970, 15, 143-156. [CrossRef]

5. Pener, M.P.; Simpson, S.J. Locust phase polyphenism: An update. Adv. Insect Physiol. 2009, 36, 1-272.

6. Wang, X.; Kang, L. Molecular mechanisms of phase change in locusts. Annu. Rev. Entomol. 2014, 59, $225-244$. [CrossRef]

7. Zera, A.J. Wing polymorphism in Gryllus (Orthoptera: Gryllidae): Proximate endocrine, energetic and biochemical mechanisms underlying morph specialization for flight vs. reproduction. In Phenotypic Plasticity of Insects: Mechanisms and Consequences, 1st ed.; Whitman, D.W., Ananthakrishnan, T.N., Eds.; CRC Press: Boca Raton, FL, USA, 2009; pp. 609-653.

8. Zera, A.J.; Denno, R.F. Physiology and ecology of dispersal polymorphism in insects. Annu. Rev. Entomol. 1997, 42, 207-230. [CrossRef]

9. Roff, D.A. The evolution of wing dimorphism in insects. Evolution 1986, 40, 1009-1020. [CrossRef]

10. Zhang, C.X.; Brisson, J.A.; Xu, H.J. Molecular mechanisms of wing polymorphism in insects. Annu. Rev. Entomol. 2019, 64, 297-314. [CrossRef]

11. Xu, H.J.; Zhang, C.X. Insulin receptors and wing dimorphism in rice planthoppers. Philos. Trans. R. Soc. Lond. B Biol. Sci. 2017, 372, 20150489. [CrossRef]

12. Xu, H.J.; Xue, J.; Lu, B.; Zhang, X.C.; Zhuo, J.C.; He, S.F.; Ma, X.F.; Jiang, Y.Q.; Fan, H.W.; Xu, J.Y.; et al. Two insulin receptors determine alternative wing morphs in planthoppers. Nature 2015, 519, $464-467$. [CrossRef]

13. Lin, X.; Xu, Y.; Jiang, J.; Lavine, M.; Lavine, L.C. Host quality induces phenotypic plasticity in a wing polyphenic insect. Proc. Natl. Acad. Sci. USA 2018, 115, 7563-7568. [CrossRef]

14. Lin, X.; Yao, Y.; Wang, B.; Lavine, M.D.; Lavine, L.C. FOXO links wing form polyphenism and wound healing in the brown planthopper, Nilaparvata lugens. Insect Biochem Mol. Biol. 2016, 70, 24-31. [CrossRef] [PubMed]

15. Liu, F.; Li, X.; Zhao, M.; Guo, M.; Han, K.; Dong, X.; Zhao, J.; Cai, W.; Zhang, Q.; Hua, H. Ultrabithorax is a key regulator for the dimorphism of wings, a main cause for the outbreak of planthoppers in rice. Natl. Sci. Rev. 2020, 7, 1181-1189. [CrossRef]

16. Iwanaga, K.; Tojo, S. Effects of juvenile-hormone and rearing density on wing dimorphism and oocyte development in the brown planthopper, Nilaparvata lugens. J. Insect Physiol. 1986, 32, 585-590. [CrossRef]

17. Vellichirammal, N.N.; Madayiputhiya, N.; Brisson, J.A. The genomewide transcriptional response underlying the pea aphid wing polyphenism. Mol. Ecol. 2016, 25, 4146-4160. [CrossRef] [PubMed]

18. Vellichirammal, N.N.; Gupta, P.; Hall, T.A.; Brisson, J.A. Ecdysone signaling underlies the pea aphid transgenerational wing polyphenism. Proc. Natl. Acad. Sci. USA 2017, 114, 1419-1423. [CrossRef]

19. Zhang, C.; Liu, X.D. Transcriptomic analysis suggests genes expressed stage-independently and stage-dependently modulating the wing dimorphism of the brown planthopper. Genes 2019, 11, 19. [CrossRef]

20. Bartel, D.P. Metazoan microRNAs. Cell 2018, 173, 20-51. [CrossRef] 
21. Ambros, V. The functions of animal microRNAs. Nature 2004, 431, 350-355. [CrossRef]

22. Bartel, D.P. MicroRNAs: Genomics, biogenesis, mechanism, and function. Cell 2004, 116, 281-297. [CrossRef]

23. Bartel, D.P. MicroRNAs: Target recognition and regulatory functions. Cell 2009, 136, 215-233. [CrossRef]

24. Kloosterman, W.P.; Plasterk, R.H.A. The diverse functions of microRNAs in animal development and disease. Dev. Cell 2006, 11, 441-450. [CrossRef] [PubMed]

25. Pillai, R.S. MicroRNA function: Multiple mechanisms for a tiny rna? RNA 2005, 11, 1753-1761. [CrossRef] [PubMed]

26. Vasudevan, S.; Tong, Y.C.; Steitz, J.A. Switching from repression to activation: MicroRNAs can up-regulate translation. Science 2007, 318, 1931-1934. [CrossRef] [PubMed]

27. He, K.; Sun, Y.; Xiao, H.; Ge, C.; Li, F.; Han, Z. Multiple miRNAs jointly regulate the biosynthesis of ecdysteroid in the holometabolous insects, Chilo Suppressalis. RNA 2017, 23, 1817-1833. [CrossRef]

28. Song, J.S.; Zhou, S.T. Post-transcriptional regulation of insect metamorphosis and oogenesis. Cell Mol. Life Sci. 2020, 77, 1893-1909. [CrossRef] [PubMed]

29. Yang, M.; Wang, Y.; Jiang, F.; Song, T.; Wang, H.; Liu, Q.; Zhang, J.; Zhang, J.; Kang, L. miR-71 and miR-263 jointly regulate target genes chitin synthase and chitinase to control locust molting. PLoS Genet. 2016, 12, e1006257. [CrossRef]

30. Yang, M.; Wei, Y.; Jiang, F.; Wang, Y.; Guo, X.; He, J.; Kang, L. MicroRNA-133 inhibits behavioral aggregation by controlling dopamine synthesis in locusts. PLoS Genet. 2014, 10, e1004206. [CrossRef]

31. Shang, F.; Niu, J.Z.B.; Ding, Y.; Zhang, W.; Wei, D.D.; Wei, D.; Jiang, H.B.; Wang, J.J. The miR-9b microRNA mediates dimorphism and development of wing in aphids. Proc. Natl. Acad. Sci. USA 2020, 117, 8404-8409. [CrossRef]

32. Ye, X.; Xu, L.; Li, X.; He, K.; Hua, H.; Cao, Z.; Xu, J.; Ye, W.; Zhang, J.; Yuan, Z.; et al. miR-34 modulates wing polyphenism in planthopper. PLoS Genet. 2019, 15, e1008235. [CrossRef]

33. Mackowiak, S.D. Identification of novel and known miRNAs in deep-sequencing data with miRDeep2. Curr. Protoc. Bioinformatics 2011, 36. [CrossRef]

34. Friedlander, M.R.; Mackowiak, S.D.; Li, N.; Chen, W.; Rajewsky, N. miRDeep2 accurately identifies known and hundreds of novel microRNA genes in seven animal clades. Nucleic Acids Res. 2012, 40,37-52. [CrossRef] [PubMed]

35. Guerra-Assuncao, J.A.; Enright, A.J. MapMi: Automated mapping of microRNA loci. BMC Bioinf. 2010, 11, 133. [CrossRef] [PubMed]

36. Chen, Q.; Lu, L.; Hua, H.; Zhou, F.; Lu, L.; Lin, Y. Characterization and comparative analysis of small RNAs in three small RNA libraries of the brown planthopper (Nilaparvata lugens). PLoS ONE 2012, 7, e32860. [CrossRef] [PubMed]

37. Wu, Y.; Lv, W.; Hu, L.; Rao, W.; Zeng, Y.; Zhu, L.; He, Y.; He, G. Identification and analysis of brown planthopper-responsive microRNAs in resistant and susceptible rice plants. Sci. Rep. 2017, 7, 8712. [CrossRef] [PubMed]

38. Love, M.I.; Huber, W.; Anders, S. Moderated estimation of fold change and dispersion for RNA-seq data with DESeq2. Genome Biol. 2014, 15, 550. [CrossRef] [PubMed]

39. John, B.; Enright, A.J.; Aravin, A.; Tuschl, T.; Sander, C.; Marks, D.S. Human microRNA targets. PLoS Biol. 2004, 2, e363. [CrossRef] [PubMed]

40. Agarwal, V.; Bell, G.W.; Nam, J.W.; Bartel, D.P. Predicting effective microRNA target sites in mammalian mRNAs. Elife 2015, 4, e05005. [CrossRef]

41. Rehmsmeier, M.; Steffen, P.; Hochsmann, M.; Giegerich, R. Fast and effective prediction of microRNA/target duplexes. RNA 2004, 10, 1507-1517. [CrossRef]

42. Kruger, J.; Rehmsmeier, M. RNAhybrid: microRNA target prediction easy, fast and flexible. Nucleic Acids Res. 2006, 34, W451-W454. [CrossRef]

43. Kertesz, M.; Iovino, N.; Unnerstall, U.; Gaul, U.; Segal, E. The role of site accessibility in microRNA target recognition. Nat. Genet. 2007, 39, 1278-1284. [CrossRef]

44. Bennett, F.C.; Harvey, K.F. Fat cadherin modulates organ size in Drosophila via the salvador/warts/hippo signaling pathway. Curr. Biol. 2006, 16, 2101-2110. [CrossRef] [PubMed]

45. Neto-Silva, R.M.; Wells, B.S.; Johnston, L.A. Mechanisms of growth and homeostasis in the Drosophila wing. Annu. Rev. Cell Dev. Biol. 2009, 25, 197-220. [CrossRef] [PubMed] 
46. Rogulja, D.; Rauskolb, C.; Irvine, K.D. Morphogen control of wing growth through the Fat signaling pathway. Dev. Cell 2008, 15, 309-321. [CrossRef]

47. Liu, S.; Li, K.; Gao, Y.; Liu, X.; Chen, W.; Ge, W.; Feng, Q.; Palli, S.R.; Li, S. Antagonistic actions of juvenile hormone and 20-hydroxyecdysone within the ring gland determine developmental transitions in Drosophila. Proc. Natl. Acad. Sci. USA 2018, 115, 139-144. [CrossRef]

48. Dye, N.A.; Popovic, M.; Spannl, S.; Etournay, R.; Kainmuller, D.; Ghosh, S.; Myers, E.W.; Julicher, F.; Eaton, S. Cell dynamics underlying oriented growth of the Drosophila wing imaginal disc. Development 2017, 144, 4406-4421. [CrossRef] [PubMed]

49. Zera, A.J. Evolutionary endocrinology of hormonal rhythms: Juvenile hormone titer circadian polymorphism in Gryllus firmus. Integr. Comp. Biol. 2016, 56, 159-170. [CrossRef] [PubMed]

50. LEES, A.D. Action of juvenile hormone mimics on the regulation of larval-adult and alary polymorphism in aphids. Nature 1977, 267, 46-48. [CrossRef]

51. Li, F.C.; Gu, Z.Y.; Wang, B.B.; Xie, Y.; Ma, L.; Xu, K.Z.; Ni, M.; Zhang, H.; Shen, W.D.; Li, B. Effects of the biosynthesis and signaling pathway of ecdysterone on silkworm (Bombyx mori) following exposure to titanium dioxide nanoparticles. J. Chem. Ecol. 2014, 40, 913-922. [CrossRef]

52. Stanley-Samuelson, D.W.; Jurenka, R.A.; Cripp, C.; Blomquist, G.J.; de Renobal, M. Fatty acids in insects: Composition, metabolrsm, and biological significance. Arch. Insect Biochem. Physiol. 1988, 9, 1-33. [CrossRef]

53. Gao, X.K.; Luo, J.Y.; Zhu, X.Z.; Wang, L.; Ji, J.C.; Zhang, L.J.; Zhang, S.; Cui, J.J. Growth and fatty acid metabolism of Aphis gossypii parasitized by the parasitic wasp Lysiphlebia japonica. J. Agr. Food Chem. 2019, 67, 8756-8765. [CrossRef]

54. Richhariya, S.; Jayakumar, S.; Sukumar, S.K.; Hasan, G. dSTIM- and Ral/exocyst-mediated synaptic release from pupal dopaminergic neurons sustains Drosophila flight. Eneuro 2018, 5, e0455-17. [CrossRef] [PubMed]

55. Pepperl, J.; Reim, G.; Luthi, U.; Kaech, A.; Hausmann, G.; Basler, K. Sphingolipid depletion impairs endocytic traffic and inhibits wingless signaling. Mech. Develop. 2013, 130, 493-505. [CrossRef] [PubMed]

56. Herr, D.R.; Fyrst, H.; Phan, V.; Heinecke, K.; Georges, R.; Harris, G.L.; Saba, J.D. Sply regulation of sphingolipid signaling molecules is essential for Drosophila development. Development 2003, 130, 2443-2453. [CrossRef] [PubMed]

57. Yang, Q.; Bao, Z.; Yang, M.; Shen, Y.; Zhang, X.; Yue, B.; Meng, Y.; Fan, Z. Identification and characterization of microRNAs in American cockroach (Periplaneta americana). Gene 2020, 743, 144610. [CrossRef] [PubMed]

58. Leevers, S.J.; Weinkove, D.; MacDougall, L.K.; Hafen, E.; Waterfield, M.D. The Drosophila phosphoinositide 3-kinase Dp110 promotes cell growth. Embo. J. 1996, 15, 6584-6594. [CrossRef] [PubMed]

59. Morooka, S.; Tojo, S. Maintenance and selection of strains exhibiting specific wing form and body color under high-density conditions in the brown planthopper, Nilaparvata lugens (Homoptera, Delphacidae). Appl. Entomol. Zool. 1992, 27, 445-454. [CrossRef]

60. Kozomara, A.; Griffiths-Jones, S. miRBase: Annotating high confidence microRNAs using deep sequencing data. Nucleic Acids Res. 2014, 42, D68-D73. [CrossRef]

61. Ma, W.H.; Xu, L.; Hua, H.X.; Chen, M.Y.; Guo, M.J.; He, K.; Zhao, J.; Li, F. Chromosomal-level genomes of three rice planthoppers provide new insights into sex chromosome evolution. Mol. Ecol. Resour. 2020. [CrossRef]

62. Yin, C.; Shen, G.; Guo, D.; Wang, S.; Ma, X.; Xiao, H.; Liu, J.; Zhang, Z.; Liu, Y.; Zhang, Y.; et al. InsectBase: A resource for insect genomes and transcriptomes. Nucleic Acids Res. 2016, 44, D801-D807. [CrossRef]

63. Conesa, A.; Gotz, S.; Garcia-Gomez, J.M.; Terol, J.; Talon, M.; Robles, M. Blast2GO: A universal tool for annotation, visualization and analysis in functional genomics research. Bioinformatics 2005, 21, 3674-3676. [CrossRef]

Publisher's Note: MDPI stays neutral with regard to jurisdictional claims in published maps and institutional affiliations. 